Urologe 2012·51:783

DOI 10.1007/s00120-012-2895-5

Online publiziert: 11. Juni 2012

(c) Springer-Verlag 2012

D. Jocham ${ }^{1} \cdot$ M. Stöckle ${ }^{2} \cdot$ M. Sommerauer ${ }^{1}$

${ }^{1}$ Klinik und Poliklinik für Urologie, Universitätsklinikum Schleswig-Holstein (UK-SH), Campus Lübeck, Lübeck

${ }^{2}$ Klinik für Urologie und Kinderurologie, Universitätsklinikum des Saarlandes Homburg/Saar, Homburg/Saar

\title{
Aspekte des Harnblasenkarzinoms
}

Blasentumoren mittels unterschiedlicher Verfahren. Hierdurch soll eine exaktere histologische Begutachtung ermöglicht werden, bei verbessertet Hämostase und Vermeidung einer Tumorzellstreuung. Insgesamt handelt es sich aber zur Zeit um ein experimentelles Verfahren, weil u. a. keine onkologischen Langzeitdaten verfügbar sind.

Die Rolle der perioperativen Chemotherapie wird aktuell kontrovers diskutiert. Mehrere Arbeiten konnten einen deutlichen Vorteil der adjuvanten Chemotherapie - insbesondere bei Vorliegen von Lymphknotenmetastasen - zeigen. Trotzdem wird die adjuvante Chemotherapie in den aktuellen EAU-Guidelines aufgrund der dünnen Datenlage nur unter Studienbedingungen empfohlen. Frau Prof. Junker et al. liefern eine fundierte Übersicht über neue prognostische und prädiktive Marker, die potentiell in der Zukunft die Option bieten, eine individuelle, risikoadaptierte Therapie durchzuführen.

Die zunehmende Lebenserwartung wird uns als Urologen in Zukunft noch häufiger mit der Problematik auch fortgeschrittener Urothelkarzinome bei älteren Patienten konfrontieren, bei denen man sich häufig zurückhaltend mit radikalchirurgischen Maßnahmen verhält. Rink et al. befassen sich in ihrem interessanten Artikel mit diesem klinisch sehr wichtigen Thema und können anhand der aktuellen Literatur belegen, dass prinzipiell auch bei älteren Patienten mit fortgeschrittenen Tumoren eine Zystektomie anderen Therapieoptionen überlegen ist und bei entsprechender Patientenselektion die perioperative Morbidität und Mortalität mit der jüngerer Patienten vergleichbar ist. Prof. Heidenreich stellt die möglichen Indikationen für eine radikale, prostataschonende Zystektomie vor. Bei dezidierter Patientenselektion ist nach Auswertung der verfügbaren Literatur ein im Vergleich zur radikalen Zystoprostatektomie gleichwertiges onkologisches Ergebnis zu erzielen, bei allerdings deutlich besserem funktionellem Ergebnis für derart behandelte Patienten.

Abschließend liefert ein Beitrag von Heck et al. aus der AUO (Arbeitsgemeinschaft Urologische Onkologie) und der IABC (Interdisziplinäre Arbeitsgruppe Blasenkarzinom) ein umfassendes Update zur systemischen Chemotherapie des Harnblasenkarzinoms.

Ziel bei der Auswahl der einzelnen Artikel für dieses Leitthemenheft war es, ein breites Spektrum thematisch abzudecken. Wir denken, dass dies gelungen ist, mit relevanten Informationen für die tägliche Praxis.

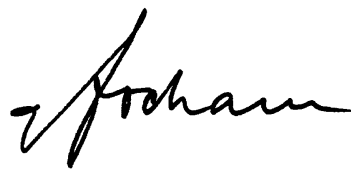

Prof.Dr. D. Jocham

\section{Korrespondenzadresse}

Prof. Dr. D. Jocham

Klinik und Poliklinik für Urologie,

Universitätsklinikum Schleswig-Holstein

(UK-SH), Campus Lübeck,

Ratzeburger Allee 160, 23538 Lübeck

dieter.jocham@uksh.de

Interessenkonflikt. Der korrespondierende Autor weist für sich und seine Koautoren auf folgende Beziehung/en hin: Prof. Dr. D. Jocham: GE Healthcare bzw. Ipsen Pharma beratend. Prof. Dr. M. Stöckle: es besteht kein Interessenkonflikt.
Kramer et al. berichten in ihrem Artikel über die En-Block-Resektion von 